\title{
Opinion as standard for all? Opposition to scientific evidence and thought
}

Wanderley Marques Bernardo ${ }^{1,2}$

1. Lecturer Professor of School of Medicine of USP; São Paulo, SP, Brasil 2. Coordinator of the Brazilian Medical Association Guidelines Program, SP, Brasil

Everyone is entitled to one or more opinions on a particular subject, and obviously, in medicine this is no different. In addition, of course the physician has the right and the autonomy to base his/her decisions and choices on his/her opinion.

That said, and philosophically speaking, the word opinion originates in the Greek (doxa), meaning, "the confused idea about reality and that opposes to the knowledge considered as true."

Scientifically speaking, opinion is the potentiated synonym of its philosophical meaning, since after thousands of years its confused ideas have been echoed thousands of times in a successful attempt to become truth. And that is also manifested in medicine.

There are many reasons for the persistency of opinion over the centuries ${ }^{1-3}$ :

- At an advanced age, it disguises as outdated, uninformed weakness and without scientific thought.

- Since it is devoid of scientific proof, it is easy and inexpensive.

- Being ambivalent, it takes advantage of the ignorance, the laziness and the haste of people and nations.

- As a cultural substitute/replacement of knowledge, it is valued as a currency and an exercise of power.
- Transactions are then fuelled by non-technical, money-intoxicated agreement policies.

- Lying, it seeks to bring down the real concepts of acquired experience, autonomy, and ethics.

- Engaging a multitude of allies around its various interests, it is dedicated to the educational dissemination of these untruths.

- Finally, in an "infinite loop", beyond persisting, it diminishes scientific knowledge as necessary truth.

- By disqualifying science as standard, it inescapably strengthens its power, by simplifying the standard for everything in as "we say so".

- In medicine, this is no different, but the consequences are, since opinion as a standard leaves patients unprotected from lies.

However, how does scientific thinking (the source of knowledge) protect the patient from decisions regarding their lives and health ${ }^{1-3}$ ?

- Establishing standards that are independent from opinions.

- Based on consistent models of clinical research.

- Refuting direct extrapolation from animal research to humans.

- Using comparisons as the main parameter.

- Comparing the conducts already in use to identify differences. 
- Using control conducts to identify if there is effect (benefit and damage).

- And if there is an effect, what is what is its size and variation?

- Reducing the number of options for the best ones to patients.

- Paving the way for innovative comparisons

- Defining the ethical boundaries between research and deployment within the system.

- Defining the roles of industry and the State in within those boundaries.

- Fighting human trials without consent.

- Building the culture of adopting only strong evidence.

- Contributing to the organization and balance of the healthcare system.

- Stimulating the establishment of equity between public and private.

- Providing patients access to those standards.

- Assisting healthcare professionals in decision-making.

- Reducing the time spent in discussing superficial clichés.

- Reducing attention on futilities with no outcomes.

- And, in exposing confused opposition, separate wheat from chaff."

In addition, forming opinions as a way of establishing standards of conduct, exposes institutions responsible for ethical-scientific zeal and care to the blow of political winds, power and corruption, taking from them the best opportunity to safeguard the rights of adequate patient care, to positively and scientifically influence healthcare professionals, and to participate actively in the organization and strategies of the system ${ }^{1-3}$.

After hundreds of years of effort and dedication of men and women to consolidate and establish current scientific thinking and strength of evidence, there is no reason why opinionated literature [traditional reports, case series or revisions (non-systematic)] should be minimally considered as standard into guiding any decision-making in healthcare. If one disagrees and wishes to impose their opinion to the detriment of science, as the Greeks in the past knew well, they might as well be confused about reality and it is pointless to argue an opinion ${ }^{1-3}$.

\section{REFERENCES}

1. Bernardo WM. Public health policies and scientific evidence. Einstein (Sao Paulo). 2017 Oct-Dec;15(4):7-10. doi: 10.1590/S1679-45082017ED4314. English, Portuguese. PubMed PMID: 29364373; PubMed Central PMCID: PMC5875176.

2. Bernardo WM. Cheers!!! Rev Assoc Med Bras (1992). 2016 Dec;62(9):809810. doi: 10.1590/1806-9282.62.09.809. PubMed PMID: 28001249.

3. Bernardo WM. Beyond the evidence: the values for the patient. Rev Assoc Med Bras (1992). 2014 Jan-Feb;60(1):1. PubMed PMID:24918842. 